Supporting Information for

\title{
Optimize the Proton Conductivity of Fe-Diphosphonates by Increasing the Relative Number of Protons and Carrier Densities
}

Xiao-Fan Jiang, ${ }^{\ddagger[a]}$ Yu-Juan Ma, ${ }^{\ddagger[a]}$ Ji-Xiang $\mathrm{Hu}^{*[a, b]}$ and Guo-Ming Wang*[a]

[a] College of Chemistry and Chemical Engineering, Qingdao University, Qingdao, Shandong 266071, China;

[b] State Key Laboratory of Fine Chemicals, Dalian University of Technology, Dalian, Liaoning 116024, China. Email: gmwang_pub@163.com

hujixianghjx@163.com 


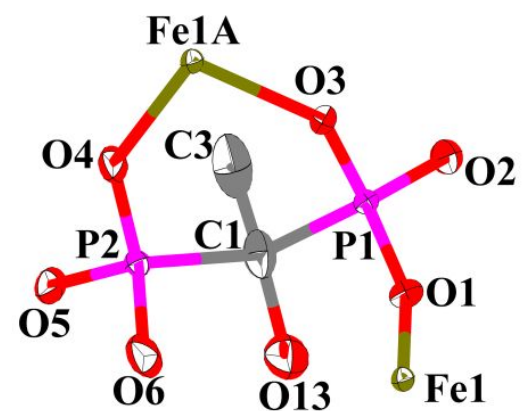

(a)

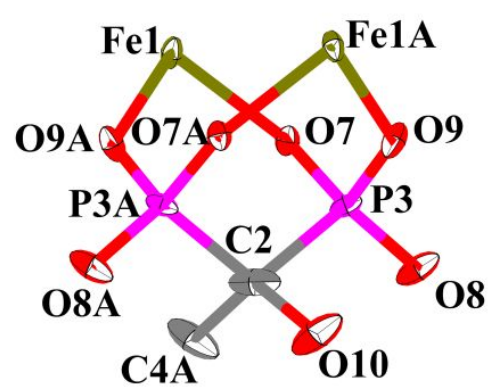

(b)

Figure S1. The coordination mode of HEDP for compound 1 (Hydrogen atoms are omitted for clarity).

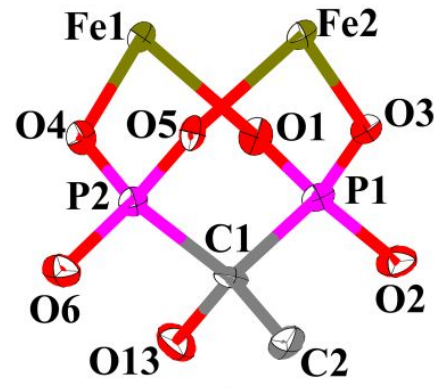

(a)

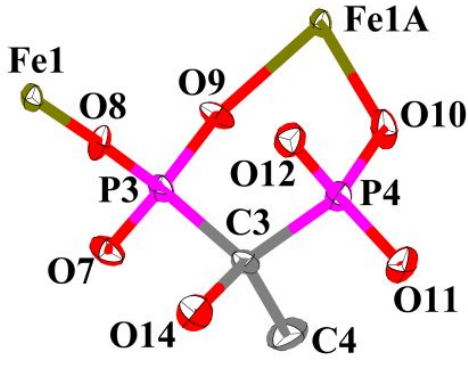

(b)

Figure S2. The coordination mode of HEDP for compound 2 (Hydrogen atoms are omitted for clarity).

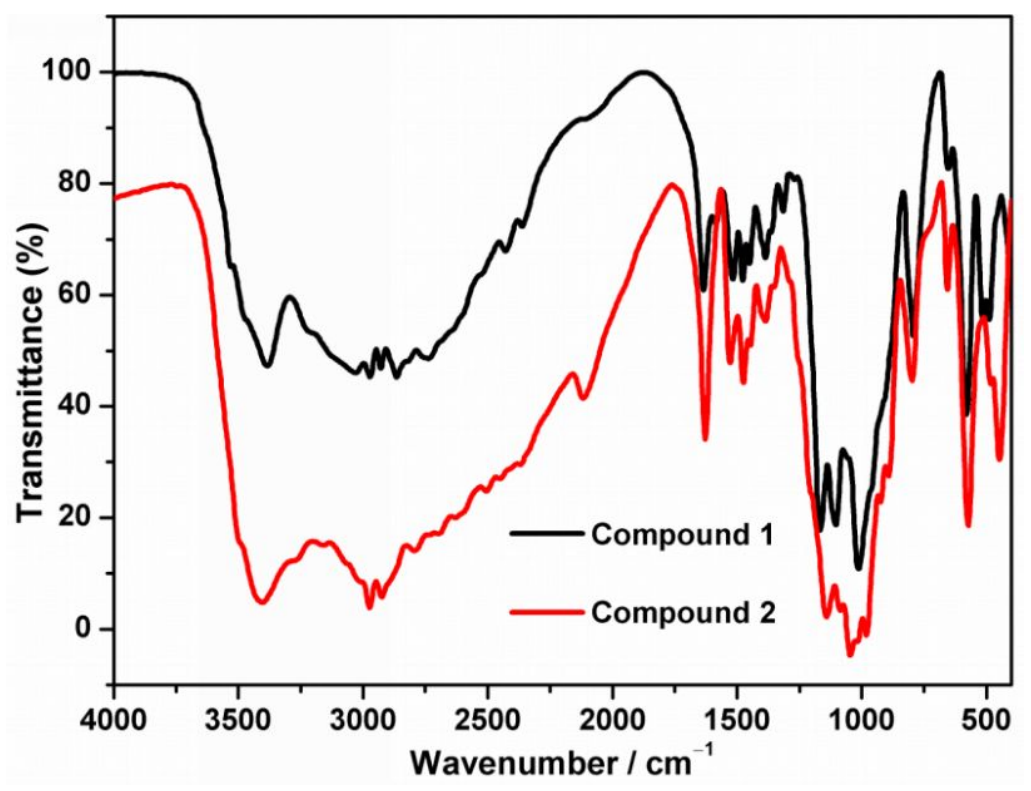

Figure S3. IR plot of 1 and 2. 


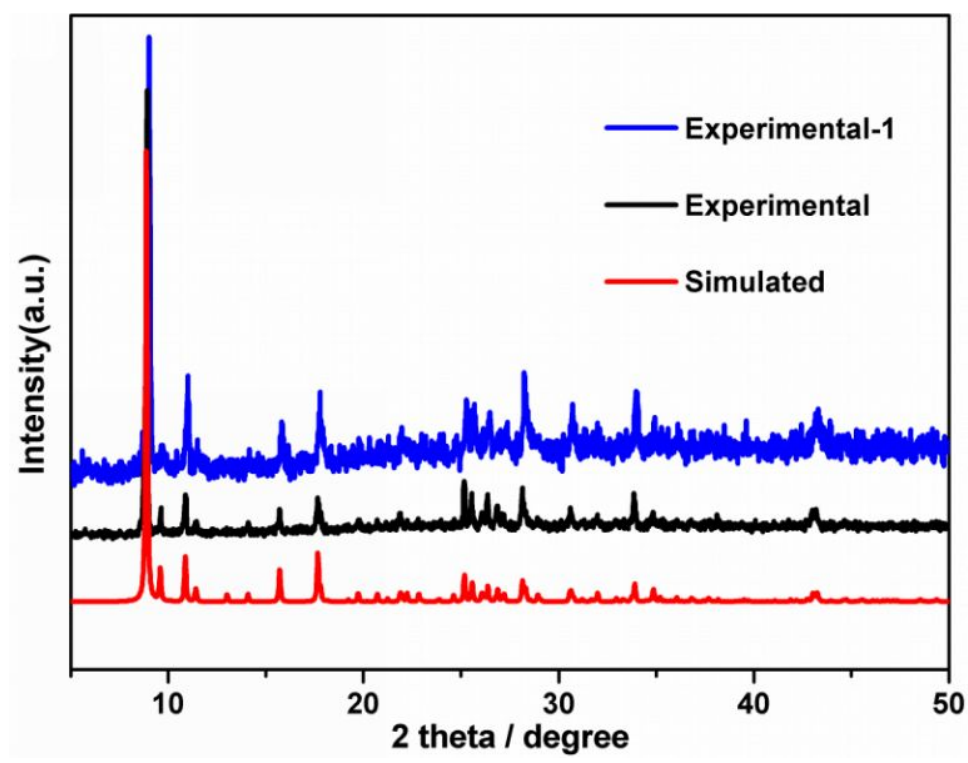

Figure S4. The PXRD plot of compound 1, the data named Experimental-1 is the sample after proton-conducting measurement.

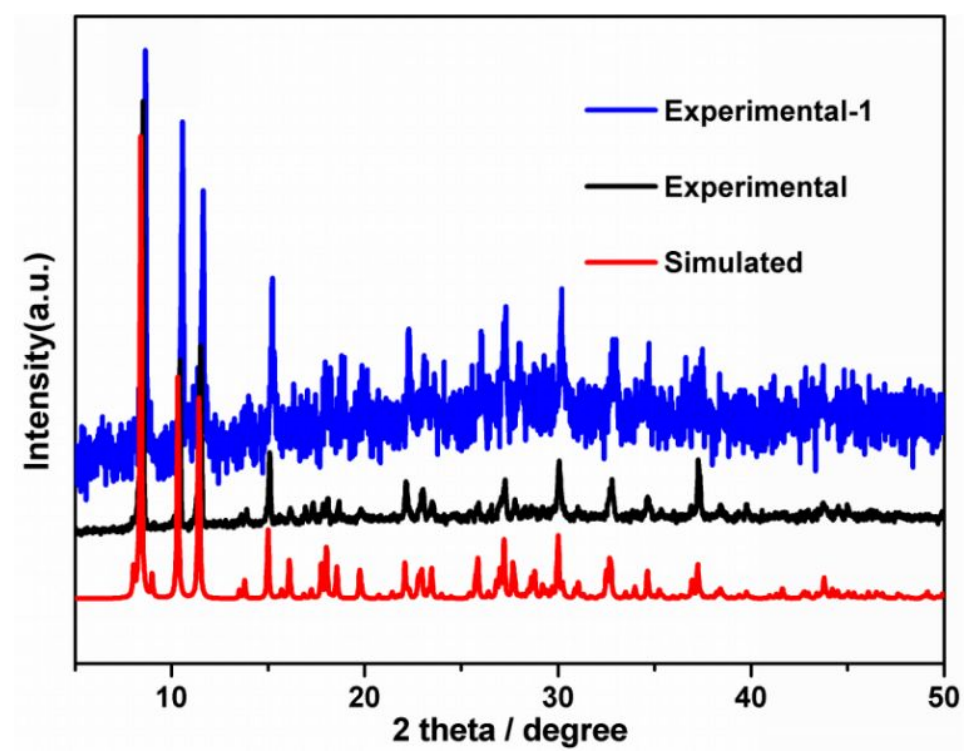

Figure S5. The PXRD plot of compound 2, the data named Experimental-1 is the sample after proton-conducting measurement. 


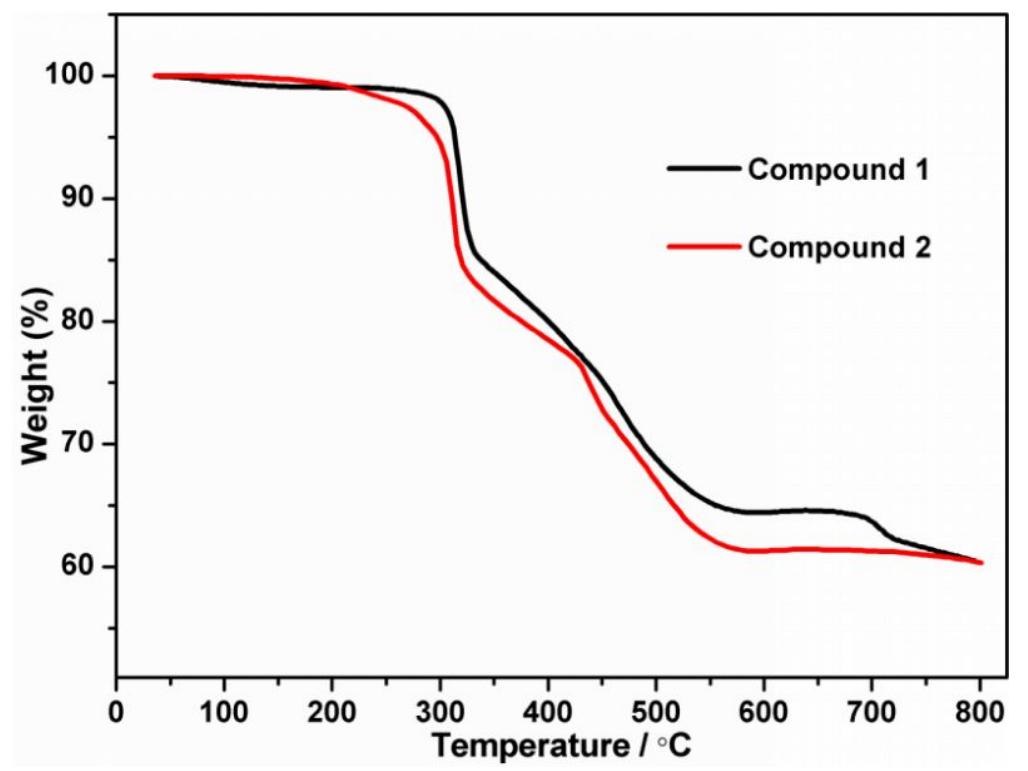

Figure S6. TGA plot of 1 and 2.

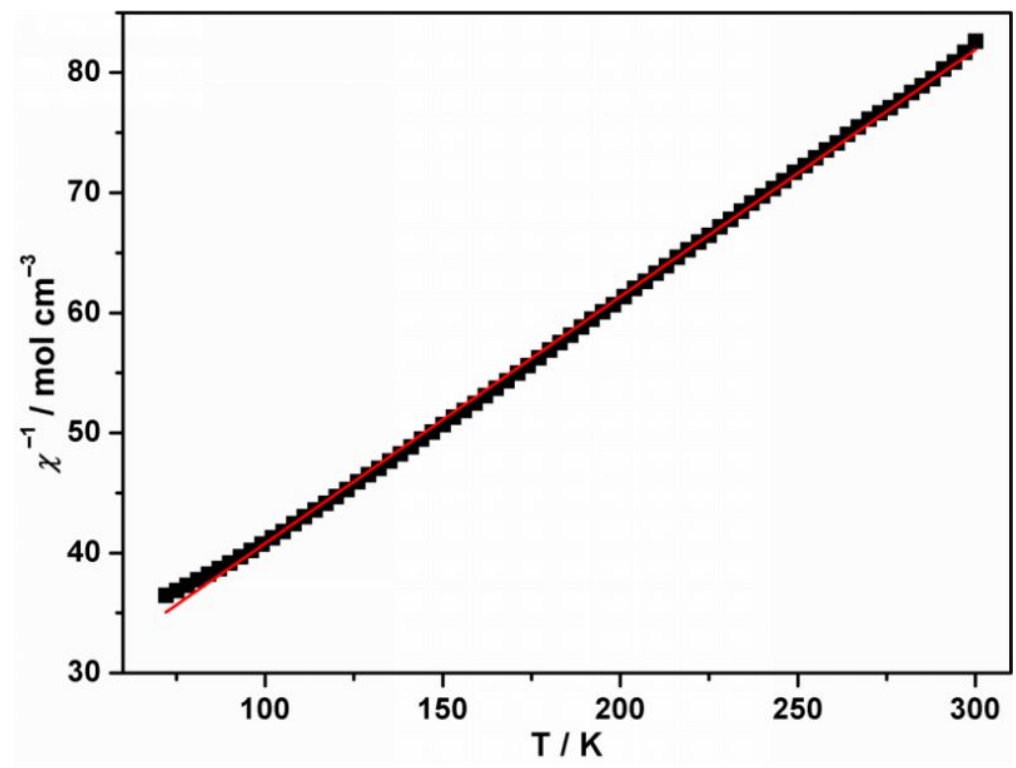

Figure S7. The $\chi^{-1}$ vs $T$ plots of $\mathbf{1}$. Solid lines represent the Curie-Weiss fitting. 


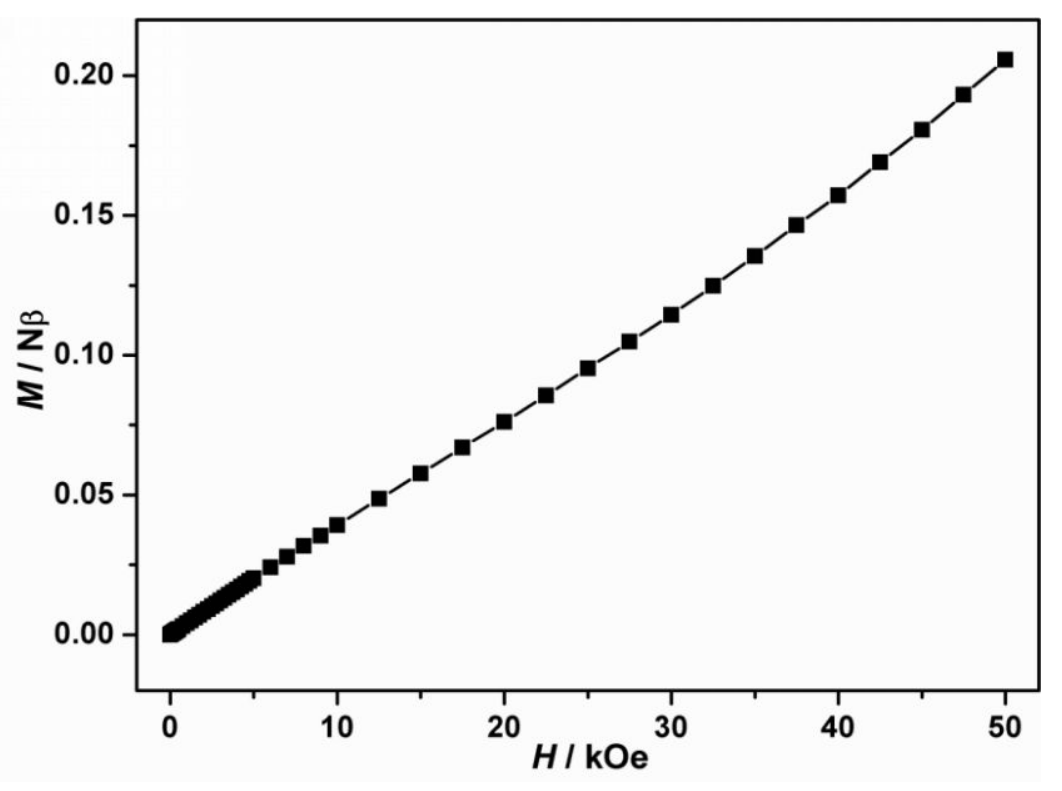

Figure S8. Isothermal magnetization curve of 1 at $2 \mathrm{~K}$.

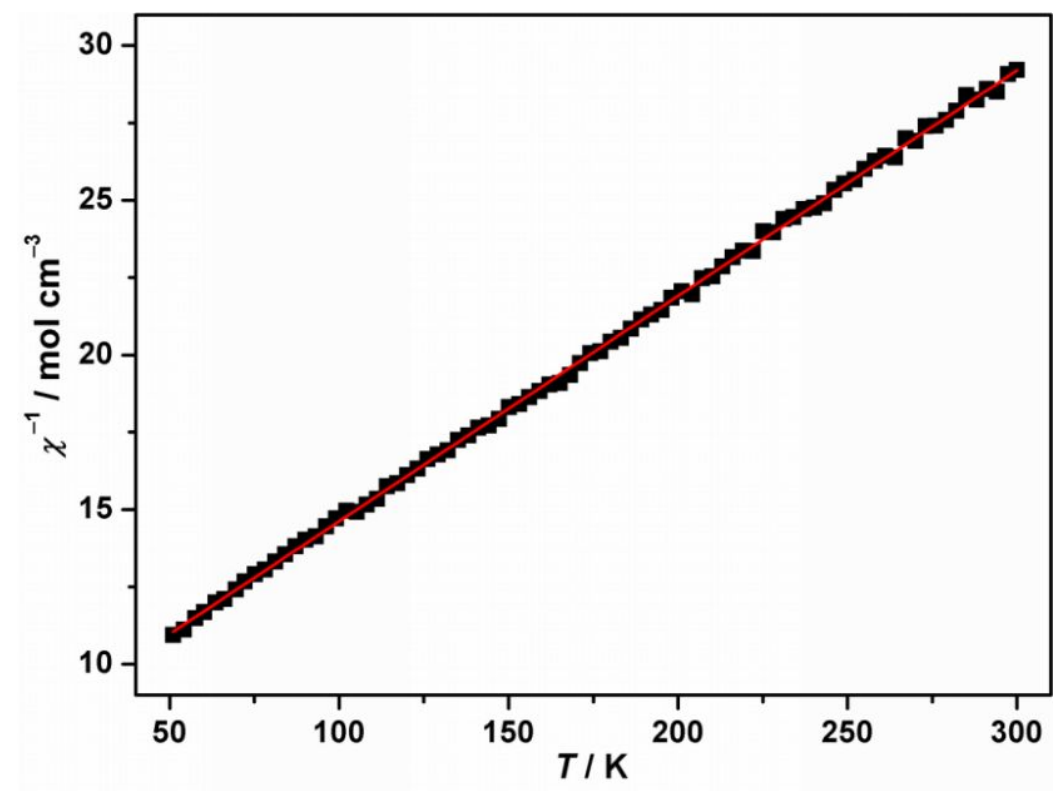

Figure S9. The $\chi^{-1} v s T$ plots of 2 . Solid lines represent the Curie-Weiss fitting. 


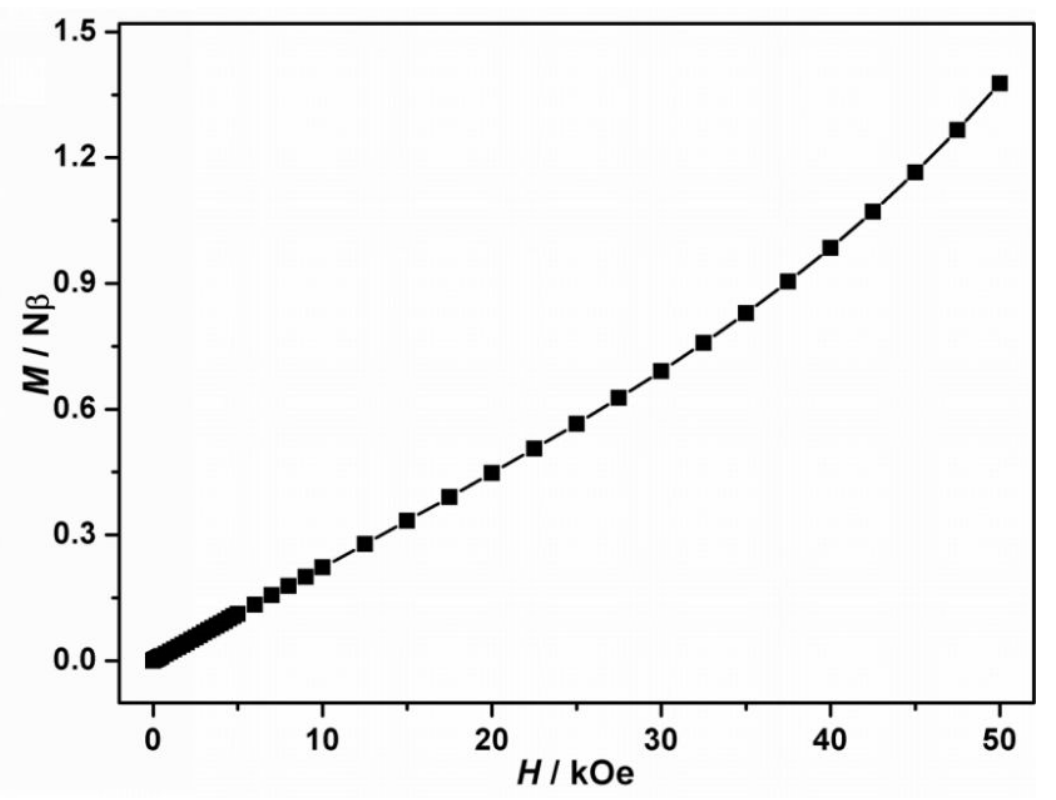

Figure S10. Isothermal magnetization curve of 2 at $2 \mathrm{~K}$.

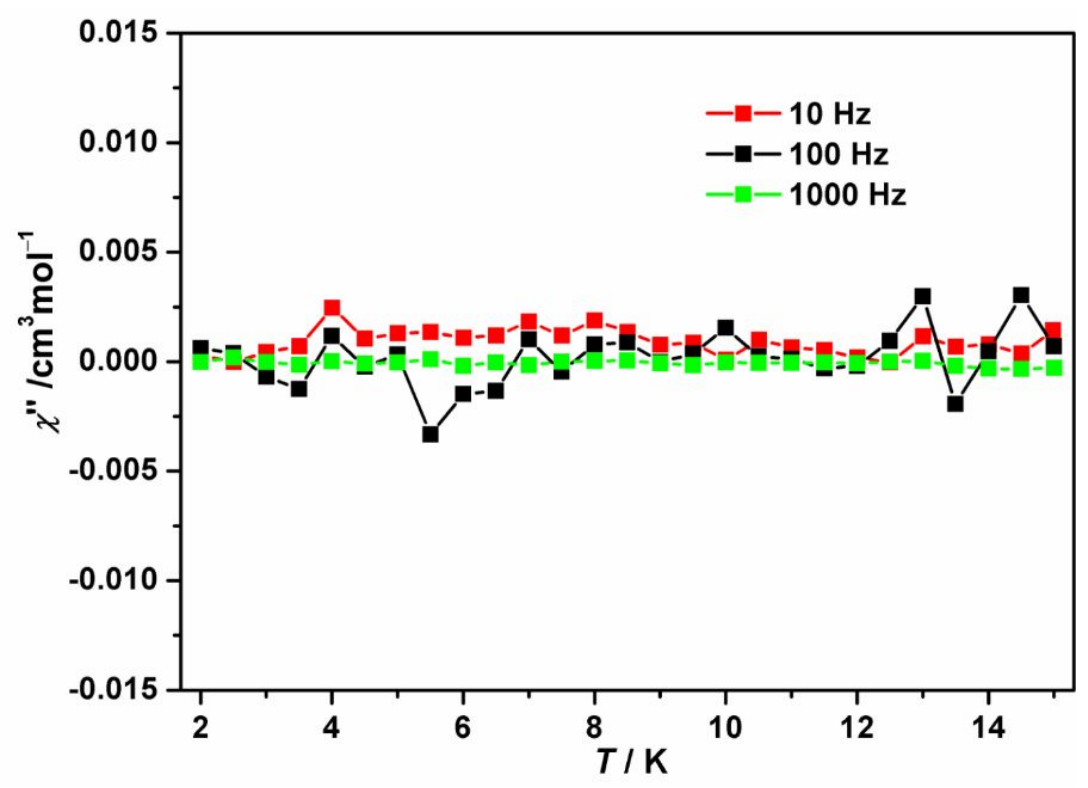

Figure S11. Temperature dependence of the out-phase components of ac magnetic susceptibility for 1 in a zero-dc and 5 Oe ac field at various ac frequencies. 


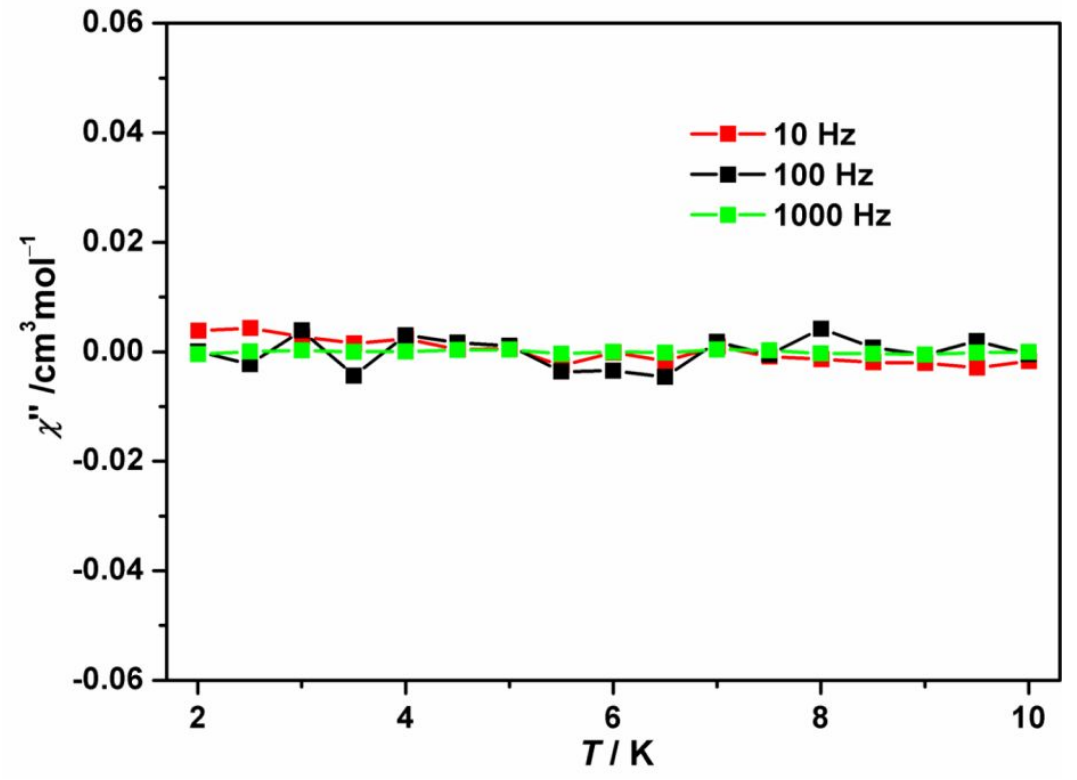

Figure S12. Temperature dependence of the out-phase components of ac magnetic susceptibility for $\mathbf{2}$ in a zero-dc and $5 \mathrm{Oe}$ ac field at various ac frequencies. 
Table S1. Selected bond lengths $(\AA)$ and angles $\left(^{\circ}\right)$ for $\mathbf{1}$

\begin{tabular}{|c|c|c|c|}
\hline $\mathrm{Fe}(1)-\mathrm{O}(1)$ & $1.962(5)$ & $\mathrm{O}(3)-\mathrm{P}(1)$ & $1.532(5)$ \\
\hline $\mathrm{Fe}(1)-\mathrm{O}(9) \# 1$ & $1.971(4)$ & $\mathrm{O}(4)-\mathrm{P}(2)$ & $1.491(5)$ \\
\hline $\mathrm{Fe}(1)-\mathrm{O}(3) \# 3$ & $1.974(5)$ & $\mathrm{O}(5)-\mathrm{P}(2)$ & $1.518(5)$ \\
\hline $\mathrm{Fe}(1)-\mathrm{O}(7)$ & $1.976(4)$ & $\mathrm{O}(6)-\mathrm{P}(2)$ & $1.532(5)$ \\
\hline $\mathrm{Fe}(1)-\mathrm{O}(4) \# 3$ & $1.992(5)$ & $\mathrm{O}(7)-\mathrm{P}(3)$ & $1.531(4)$ \\
\hline $\mathrm{Fe}(1)-\mathrm{O}(12)$ & $2.027(2)$ & $\mathrm{O}(8)-\mathrm{P}(3)$ & $1.487(5)$ \\
\hline $\mathrm{O}(1)-\mathrm{P}(1)$ & $1.506(5)$ & $\mathrm{O}(9)-\mathrm{P}(3)$ & $1.533(5)$ \\
\hline $\mathrm{O}(2)-\mathrm{P}(1)$ & $1.507(5)$ & & \\
\hline $\mathrm{O}(1)-\mathrm{Fe}(1)-\mathrm{O}(9) \# 1$ & $87.13(19)$ & $\mathrm{O}(3) \# 3-\mathrm{Fe}(1)-\mathrm{O}(12)$ & $89.11(18)$ \\
\hline $\mathrm{O}(1)-\mathrm{Fe}(1)-\mathrm{O}(3) \# 3$ & $94.5(2)$ & $\mathrm{O}(7)-\mathrm{Fe}(1)-\mathrm{O}(12)$ & $86.38(16)$ \\
\hline $\mathrm{O}(9) \# 1-\mathrm{Fe}(1)-\mathrm{O}(3) \# 3$ & $89.8(2)$ & $\mathrm{O}(4) \# 3-\mathrm{Fe}(1)-\mathrm{O}(12)$ & $90.8(2)$ \\
\hline $\mathrm{O}(1)-\mathrm{Fe}(1)-\mathrm{O}(7)$ & $90.12(19)$ & $\mathrm{O}(1)-\mathrm{P}(1)-\mathrm{O}(2)$ & $112.1(3)$ \\
\hline $\mathrm{O}(9) \# 1-\mathrm{Fe}(1)-\mathrm{O}(7)$ & $92.52(19)$ & $\mathrm{O}(1)-\mathrm{P}(1)-\mathrm{O}(3)$ & $111.2(3)$ \\
\hline $\mathrm{O}(3) \# 3-\mathrm{Fe}(1)-\mathrm{O}(7)$ & 174.92(19) & $\mathrm{O}(2)-\mathrm{P}(1)-\mathrm{O}(3)$ & $111.2(3)$ \\
\hline $\mathrm{O}(1)-\mathrm{Fe}(1)-\mathrm{O}(4) \# 3$ & $92.9(2)$ & $\mathrm{O}(4)-\mathrm{P}(2)-\mathrm{O}(5)$ & $107.9(3)$ \\
\hline $\mathrm{O}(9) \# 1-\mathrm{Fe}(1)-\mathrm{O}(4) \# 3$ & $179.9(2)$ & $\mathrm{O}(4)-\mathrm{P}(2)-\mathrm{O}(6)$ & $113.3(4)$ \\
\hline $\mathrm{O}(3) \# 3-\mathrm{Fe}(1)-\mathrm{O}(4) \# 3$ & $90.3(2)$ & $\mathrm{O}(5)-\mathrm{P}(2)-\mathrm{O}(6)$ & $106.8(3)$ \\
\hline $\mathrm{O}(7)-\mathrm{Fe}(1)-\mathrm{O}(4) \# 3$ & $87.40(19)$ & $\mathrm{O}(8)-\mathrm{P}(3)-\mathrm{O}(7)$ & $110.1(3)$ \\
\hline $\mathrm{O}(1)-\mathrm{Fe}(1)-\mathrm{O}(12)$ & $174.8(2)$ & $\mathrm{O}(8)-\mathrm{P}(3)-\mathrm{O}(9)$ & $113.5(3)$ \\
\hline $\mathrm{O}(9) \# 1-\mathrm{Fe}(1)-\mathrm{O}(12)$ & $89.14(16)$ & $\mathrm{O}(7)-\mathrm{P}(3)-\mathrm{O}(9)$ & $109.7(3)$ \\
\hline
\end{tabular}

Symmetry codes: \#1: $-\mathrm{x}+2, \mathrm{y},-\mathrm{z}+3 / 2 ; \# 2:-\mathrm{x}+1 / 2,-\mathrm{y}+3 / 2,-\mathrm{z}+2 ; \# 3:-x+2,-y+1,-z+2$. 
Table S2. Selected bond lengths $(\AA)$ and angles $\left(^{\circ}\right)$ for 2

\begin{tabular}{|c|c|c|c|}
\hline $\mathrm{O}(16)-\mathrm{Fe}(2)$ & $1.974(4)$ & $\mathrm{O}(2)-\mathrm{P}(1)$ & $1.507(5)$ \\
\hline $\mathrm{O}(16)-\mathrm{Fe}(1)$ & $2.037(4)$ & $\mathrm{O}(3)-\mathrm{P}(1)$ & $1.535(4)$ \\
\hline $\mathrm{Fe}(1)-\mathrm{O}(8)$ & $2.007(4)$ & $\mathrm{O}(4)-\mathrm{P}(2)$ & $1.529(5)$ \\
\hline $\mathrm{Fe}(1)-\mathrm{O}(9) \# 1$ & $2.031(5)$ & $\mathrm{O}(5)-\mathrm{P}(2)$ & $1.554(4)$ \\
\hline $\mathrm{Fe}(1)-\mathrm{O}(4)$ & $2.034(5)$ & $\mathrm{O}(6)-\mathrm{P}(2)$ & $1.486(5)$ \\
\hline $\mathrm{Fe}(1)-\mathrm{O}(1)$ & $2.048(5)$ & $\mathrm{O}(7)-\mathrm{P}(3)$ & $1.492(6)$ \\
\hline $\mathrm{Fe}(1)-\mathrm{O}(10) \# 1$ & $2.086(5)$ & $\mathrm{O}(8)-\mathrm{P}(3)$ & $1.507(4)$ \\
\hline $\mathrm{Fe}(2)-\mathrm{O}(5) \# 2$ & $1.987(5)$ & $\mathrm{O}(9)-\mathrm{P}(3)$ & $1.531(4)$ \\
\hline $\mathrm{Fe}(2)-\mathrm{O}(5)$ & $1.987(5)$ & $\mathrm{O}(10)-\mathrm{P}(4)$ & $1.519(5)$ \\
\hline $\mathrm{Fe}(2)-\mathrm{O}(3)$ & $2.005(4)$ & $\mathrm{O}(11)-\mathrm{P}(4)$ & $1.489(5)$ \\
\hline $\mathrm{Fe}(2)-\mathrm{O}(3) \# 2$ & $2.005(4)$ & $\mathrm{O}(12)-\mathrm{P}(4)$ & $1.564(5)$ \\
\hline $\mathrm{O}(1)-\mathrm{P}(1)$ & $1.523(5)$ & & \\
\hline $\mathrm{O}(8)-\mathrm{Fe}(1)-\mathrm{O}(9) \# 1$ & $92.52(19)$ & $\mathrm{O}(16) \# 2-\mathrm{Fe}(2)-\mathrm{O}(3)$ & $89.63(17)$ \\
\hline $\mathrm{O}(8)-\mathrm{Fe}(1)-\mathrm{O}(4)$ & $92.16(19)$ & $\mathrm{O}(16)-\mathrm{Fe}(2)-\mathrm{O}(3)$ & $90.37(17)$ \\
\hline $\mathrm{O}(9) \# 1-\mathrm{Fe}(1)-\mathrm{O}(4)$ & $175.26(17)$ & $\mathrm{O}(5) \# 2-\mathrm{Fe}(2)-\mathrm{O}(3)$ & $88.23(19)$ \\
\hline $\mathrm{O}(8)-\mathrm{Fe}(1)-\mathrm{O}(16)$ & $174.33(18)$ & $\mathrm{O}(5)-\mathrm{Fe}(2)-\mathrm{O}(3)$ & $91.77(19)$ \\
\hline $\mathrm{O}(9) \# 1-\mathrm{Fe}(1)-\mathrm{O}(16)$ & $85.85(17)$ & $\mathrm{O}(16) \# 2-\mathrm{Fe}(2)-\mathrm{O}(3) \# 2$ & $90.37(17)$ \\
\hline $\mathrm{O}(4)-\mathrm{Fe}(1)-\mathrm{O}(16)$ & $89.42(17)$ & $\mathrm{O}(16)-\mathrm{Fe}(2)-\mathrm{O}(3) \# 2$ & $89.63(17)$ \\
\hline $\mathrm{O}(8)-\mathrm{Fe}(1)-\mathrm{O}(1)$ & $93.85(19)$ & $\mathrm{O}(5) \# 2-\mathrm{Fe}(2)-\mathrm{O}(3) \# 2$ & $91.77(19)$ \\
\hline $\mathrm{O}(9) \# 1-\mathrm{Fe}(1)-\mathrm{O}(1)$ & $90.9(2)$ & $\mathrm{O}(5)-\mathrm{Fe}(2)-\mathrm{O}(3) \# 2$ & $88.23(19)$ \\
\hline $\mathrm{O}(4)-\mathrm{Fe}(1)-\mathrm{O}(1)$ & $89.59(19)$ & $\mathrm{O}(3)-\mathrm{Fe}(2)-\mathrm{O}(3) \# 2$ & 180.0 \\
\hline $\mathrm{O}(16)-\mathrm{Fe}(1)-\mathrm{O}(1)$ & $91.61(17)$ & $\mathrm{O}(2)-\mathrm{P}(1)-\mathrm{O}(1)$ & $112.5(3)$ \\
\hline $\mathrm{O}(8)-\mathrm{Fe}(1)-\mathrm{O}(10) \# 1$ & $90.2(2)$ & $\mathrm{O}(2)-\mathrm{P}(1)-\mathrm{O}(3)$ & $111.1(3)$ \\
\hline $\mathrm{O}(9) \# 1-\mathrm{Fe}(1)-\mathrm{O}(10) \# 1$ & $88.0(2)$ & $\mathrm{O}(1)-\mathrm{P}(1)-\mathrm{O}(3)$ & $111.5(2)$ \\
\hline $\mathrm{O}(4)-\mathrm{Fe}(1)-\mathrm{O}(10) \# 1$ & $91.2(2)$ & $\mathrm{O}(6)-\mathrm{P}(2)-\mathrm{O}(4)$ & $112.1(3)$ \\
\hline $\mathrm{O}(16)-\mathrm{Fe}(1)-\mathrm{O}(10) \# 1$ & $84.33(18)$ & $\mathrm{O}(6)-\mathrm{P}(2)-\mathrm{O}(5)$ & $113.0(3)$ \\
\hline $\mathrm{O}(1)-\mathrm{Fe}(1)-\mathrm{O}(10) \# 1$ & $175.85(18)$ & $\mathrm{O}(4)-\mathrm{P}(2)-\mathrm{O}(5)$ & $110.4(2)$ \\
\hline
\end{tabular}




\begin{tabular}{lcll}
\hline $\mathrm{O}(16) \# 2-\mathrm{Fe}(2)-\mathrm{O}(16)$ & 180.0 & $\mathrm{O}(7)-\mathrm{P}(3)-\mathrm{O}(8)$ & $112.5(3)$ \\
$\mathrm{O}(16) \# 2-\mathrm{Fe}(2)-\mathrm{O}(5) \# 2$ & $90.82(17)$ & $\mathrm{O}(7)-\mathrm{P}(3)-\mathrm{O}(9)$ & $110.7(3)$ \\
$\mathrm{O}(16)-\mathrm{Fe}(2)-\mathrm{O}(5) \# 2$ & $89.18(17)$ & $\mathrm{O}(8)-\mathrm{P}(3)-\mathrm{O}(9)$ & $111.5(3)$ \\
$\mathrm{O}(16) \# 2-\mathrm{Fe}(2)-\mathrm{O}(5)$ & $89.18(17)$ & $\mathrm{O}(11)-\mathrm{P}(4)-\mathrm{O}(10)$ & $116.2(3)$ \\
$\mathrm{O}(16)-\mathrm{Fe}(2)-\mathrm{O}(5)$ & $90.82(17)$ & $\mathrm{O}(11)-\mathrm{P}(4)-\mathrm{O}(12)$ & $105.8(3)$ \\
$\mathrm{O}(5) \# 2-\mathrm{Fe}(2)-\mathrm{O}(5)$ & 180.0 & $\mathrm{O}(10)-\mathrm{P}(4)-\mathrm{O}(12)$ & $110.1(3)$ \\
\hline
\end{tabular}

Symmetry codes: $\# 1:-x+1,-y+2,-z ; \# 2:-x+1,-y+2,-z+1$.

Table S3. Details of Hydrogen Bond Interactions in 1 at 293K.

\begin{tabular}{|c|c|c|c|c|}
\hline $\mathrm{D}-\mathrm{H} \cdots \mathrm{A}$ & $d(\mathrm{D}-\mathrm{H})(\AA)$ & $d(\mathrm{H} \cdots \mathrm{A})(\AA)$ & $d(\mathrm{D} \cdots \mathrm{A})(\AA)$ & $\angle(\mathrm{DHA})(\mathrm{deg})$ \\
\hline O13-H13...O9_\$1 & 0.82 & 2.59 & $3.252(12)$ & 139.4 \\
\hline O13-H13...O6 & 0.82 & 2.38 & $2.912(11)$ & 123.7 \\
\hline O6-H6...O3_\$3 & 0.84 & 2.02 & $2.819(7)$ & 158.3 \\
\hline N2-H2B...O8_\$4 & 0.89 & 2.61 & $3.191(7)$ & 123.4 \\
\hline N2-H2B...O7_\$4 & 0.89 & 1.98 & $2.831(6)$ & 160.9 \\
\hline N2-H2A...O2_\$5 & 0.89 & 1.84 & $2.717(7)$ & 166.5 \\
\hline N1-H1C...O5_\$8 & 0.89 & 2.11 & $2.975(8)$ & 165.2 \\
\hline N1-H1C...O4_\$8 & 0.89 & 2.63 & $3.287(8)$ & 131.1 \\
\hline N1-H1B...O2_\$7 & 0.89 & 2.05 & $2.801(8)$ & 141.6 \\
\hline N1-H1A...O8_\$6 & 0.89 & 1.76 & $2.592(8)$ & 155.2 \\
\hline
\end{tabular}


Table S4. Details of Hydrogen Bond Interactions in 2 at 293K.

\begin{tabular}{|c|c|c|c|c|}
\hline $\mathrm{D}-\mathrm{H} \cdots \mathrm{A}$ & $d(\mathrm{D}-\mathrm{H})(\AA)$ & $d(\mathrm{H} \cdots \mathrm{A})(\AA)$ & $d(\mathrm{D} \cdots \mathrm{A})(\AA)$ & $\angle$ (DHA) (deg) \\
\hline O16-H16A...N1_\$3 & 0.89 & 2.65 & $3.096(8)$ & 112.4 \\
\hline O16-H16A...O5_\$1 & 0.89 & 1.90 & $2.780(6)$ & 168.1 \\
\hline O16-H16B...N4_\$4 & 0.89 & 2.54 & $3.096(8)$ & 121.4 \\
\hline O16-H16B...O3_\$1 & 0.89 & 1.96 & $2.805(6)$ & 158.7 \\
\hline O16-H16B...O5 & 0.89 & 2.38 & $2.821(6)$ & 110.9 \\
\hline N1-H1A...O16_\$6 & 0.89 & 2.53 & $3.096(8)$ & 122.3 \\
\hline N1-H1A...O9_\$7 & 0.89 & 2.00 & $2.860(7)$ & 163.0 \\
\hline N1-H1B...O15_\$8 & 0.89 & 1.91 & $2.785(10)$ & 165.8 \\
\hline N1-H1C...O5_\$8 & 0.89 & 1.99 & $2.845(8)$ & 160.7 \\
\hline N2-H2D...O7 & 0.89 & 1.75 & $2.601(8)$ & 160.6 \\
\hline N2-H2E...O4 & 0.89 & 1.97 & $2.819(7)$ & 158.4 \\
\hline N3-H3A...O2_\$5 & 0.89 & 2.03 & $2.901(19)$ & 165.0 \\
\hline N3-H3B...O2_\$6 & 0.89 & 1.83 & $2.709(17)$ & 170.3 \\
\hline N3A-H3A1_..O2_\$6 & 0.89 & 1.95 & $2.808(13)$ & 161.3 \\
\hline N3A-H3A2 ..O11_\$6 & 0.89 & 2.10 & $2.932(12)$ & 155.7 \\
\hline N3A-H3A2 ..O12_\$6 & 0.89 & 2.62 & $3.166(12)$ & 120.9 \\
\hline N4-H4D...O3_\$5 & 0.89 & 1.95 & $2.821(8)$ & 165.3 \\
\hline N4-H4E...O16_\$9 & 0.89 & 2.52 & $3.096(8)$ & 123.0 \\
\hline N4-H4E...O10_\$10 & 0.89 & 1.99 & $2.843(7)$ & 160.8 \\
\hline N4-H4F...O6_\$11 & 0.89 & 1.83 & $2.678(8)$ & 159.7 \\
\hline O12-H12...O9_\$2 & 0.90 & 2.04 & $2.934(7)$ & 171.1 \\
\hline O14-H14...O1 & 0.82 & 2.14 & $2.946(7)$ & 169.9 \\
\hline O15-H15C...O11_\$5 & 0.95 & 2.15 & $3.083(10)$ & 169.4 \\
\hline O15-H15D...O11_\$12 & 0.90 & 1.80 & $2.691(10)$ & 171.0 \\
\hline
\end{tabular}


Table S5. Continuous Shape Measure (CShM) analyses of geometries for Fe1 in compound $\mathbf{1}$ by SHAPE 2.0 Software

\begin{tabular}{ll}
\hline Geometry & $\mathbf{F e} 1$ \\
\hline Hexagon $\left(D_{6 h}\right)$ & 31.765 \\
Pentagonal pyramid $\left(C_{5 v}\right)$ & 27.955 \\
Octahedron $\left(O_{h}\right)$ & 0.176 \\
Trigonal prism $\left(D_{3 h}\right)$ & 15.116 \\
Johnson pentagonal pyramid $\mathrm{J} 2\left(C_{5 v}\right)$ & 31.631 \\
\hline
\end{tabular}

Table S6. Continuous Shape Measure (CShM) analyses of geometries for Fe1 and $\mathrm{Fe} 2$ in compound 2 by SHAPE 2.0 Software

\begin{tabular}{lcc}
\hline Geometry & $\mathbf{F e} 1$ & $\mathbf{F e} 2$ \\
\hline Hexagon $\left(D_{6 h}\right)$ & 32.959 & 32.294 \\
Pentagonal pyramid $\left(C_{5 v}\right)$ & 28.905 & 29.861 \\
Octahedron $\left(O_{h}\right)$ & 0.108 & 0.021 \\
Trigonal prism $\left(D_{3 h}\right)$ & 15.779 & 16.503 \\
Johnson pentagonal pyramid $\mathrm{J} 2\left(C_{5 v}\right)$ & 32.672 & 33.344 \\
\hline
\end{tabular}

